\title{
On the maximum number of spanning copies of an orientation in a tournament
}

\author{
Raphael Yuster *
}

\begin{abstract}
For an orientation $H$ with $n$ vertices, let $T(H)$ denote the maximum possible number of labeled copies of $H$ in an $n$-vertex tournament. It is easily seen that $T(H) \geq n ! / 2^{e(H)}$ as the latter is the expected number of such copies in a random tournament. For $n$ odd, let $R(H)$ denote the maximum possible number of labeled copies of $H$ in an $n$-vertex regular tournament. Adler et al. proved that, in fact, for $H=C_{n}$ the directed Hamilton cycle, $T\left(C_{n}\right) \geq(e-o(1)) n ! / 2^{n}$ and it was observed by Alon that already $R\left(C_{n}\right) \geq(e-o(1)) n ! / 2^{n}$. Similar results hold for the directed Hamilton path $P_{n}$. In other words, for the Hamilton path and cycle, the lower bound derived from the expectation argument can be improved by a constant factor. In this paper we significantly extend these results and prove that they hold for a larger family of orientations $H$ which includes all bounded degree Eulerian orientations and all bounded degree balanced orientations, as well as many others. One corollary of our method is that for any $k$-regular orientation $H$ with $n$ vertices, $T(H) \geq\left(e^{k}-o(1)\right) n ! / 2^{e(H)}$ and in fact, for $n$ odd, $R(H) \geq\left(e^{k}-o(1)\right) n ! / 2^{e(H)}$.
\end{abstract}

\section{Introduction}

All graphs and digraph considered in this paper are finite and simple. Our graph-theoretic notation is standard and follows that of [4]. An orientation is a digraph without directed cycles of length 2. Stated otherwise, an orientation $H$ of an undirected graph $\hat{H}$ is obtained by orienting each edge of $\hat{H}$. We say that $\hat{H}$ is the underlying graph of $H$. We use the notation $(u, v)$ for an edge directed from $u$ to $v$. For a vertex $v$ of an orientation its out-degree, denoted $d^{+}(v)$, is the number of edges directed from $v$ while its in-degree, denoted $d^{-}(v)$, is the number of edges directed to $v$. An orientation $H$ is $k$-regular if $d^{+}(v)=d^{-}(v)=k$ for all vertices $v \in V(H)$. It is Eulerian if $d^{+}(v)=d^{-}(v)$ for all vertices $v \in V(H)$, and $\hat{H}$ is connected (if connectivity of $\hat{H}$ is not assumed we say that $H$ is even). An orientation is balanced if $\left|d^{+}(v)-d^{-}(v)\right| \leq 1$ for all vertices $v \in V(H)$.

A tournament is an orientation of a complete graph. A tournament is transitive if it contains no directed cycle, or equivalently, if it is possible to order its vertices so that all edges are oriented

${ }^{*}$ Department of Mathematics, University of Haifa, Haifa 31905, Israel. Email: raphy@math.haifa.ac.il 
from left to right. We use $T_{n}$ to denote the (unique) $n$-vertex transitive tournament. A tournament with $n$ vertices is regular if $d^{+}(v)=d^{-}(v)=(n-1) / 2$ for all vertices $v \in V(H)$ (so here $n$ is odd).

For an orientation $H$ and a tournament $G$, both with the same number of vertices, let $G(H)$ denote the number of labeled copies of $H$ in $G$. Notice that each such labeled copy is a labeled spanning subgraph of $G$ and notice also that the number of unlabeled copies of $H$ in $G$ is $G(H) / \operatorname{aut}(H)$ where $\operatorname{aut}(H)$ is the number of automorphisms of $H$. Our objects of interest in this paper are $T(H)$, which denotes the maximum of $G(H)$ ranging over all tournaments with the same number of vertices of $H$, and $R(H)$, which denotes the maximum of $G(H)$ ranging over all regular tournaments with the same number of vertices of $H$ (so for $R(H)$ we assume that the number of vertices is odd).

Let $C_{n}$ denote the directed cycle on $n$ vertices and let $P_{n}$ denote the directed path on $n$ vertices. The problems of determining $T\left(C_{n}\right), R\left(C_{n}\right), T\left(P_{n}\right), R\left(P_{n}\right)$ are well-studied and fairly well-understood. The first lower bound for $T\left(P_{n}\right)$ was proved by Szele [14] who showed that $T\left(P_{n}\right) \geq n ! / 2^{n-1}$. It seems that his argument is the first application of the probabilistic method in combinatorics. Indeed, observe that $n ! / 2^{n-1}$ is the expected number of copies of $P_{n}$ in a random tournament. Thus, Szele's argument actually proves that $T(H) \geq n ! / 2^{e(H)}$ for any orientation $H$ with $n$ vertices (we call this the "expectation lower bound") and in particular, $T\left(C_{n}\right) \geq n ! / 2^{n}$, or, equivalently, since $\operatorname{aut}\left(C_{n}\right)=n$, there are tournaments which contain at least $(n-1) ! / 2^{n}$ Hamilton cycles. Szele also proved the upper bound $T\left(P_{n}\right)=O\left(n ! / 2^{0.75 n}\right)$. Many years later, this upper bound was significantly improved by Alon [2] who showed that $T\left(P_{n}\right)=O\left(n^{3 / 2} n ! / 2^{n-1}\right)$. In other words, this upper bound is only larger than the expectation lower bound by a small polynomial factor. Later, the polynomial was further improved to slightly less than $n^{5 / 4}$ by Friedgut and Kahn 9]. Both of these results apply also to $T\left(C_{n}\right)$.

The first, perhaps surprising improvement over the expectation lower bound, was obtained by Adler, Alon, and Ross [1]. They have shown, that, in fact $T\left(P_{n}\right) \geq(e-o(1)) n ! / 2^{n-1}$ and similarly, $T\left(C_{n}\right) \geq(e-o(1)) n ! / 2^{n}$. It was even more surprising that later Wormald [18] proved that the factor $e$ can be replaced with a constant larger than 2.855. His clever probabilistic construction involves a computer assisted verification. Thomassen (see [5]) asked in 1990 whether $R\left(C_{n}\right) \geq n ! / 2^{n}$, that is, whether the expectation lower bound also applies to the restricted case of regular tournaments. Alon [3] observed that the proof from [1] can, in fact, be adjusted to show that it holds also for regular tournaments, namely, $R\left(P_{n}\right) \geq(e-o(1)) n ! / 2^{n-1}$ and $R\left(C_{n}\right) \geq(e-o(1)) n ! / 2^{n}$. In fact, Wormald's proof with the improved constant 2.855 also applies to the regular setting. It was suggested in [1, 9] that $T\left(P_{n}\right)$ and $R\left(P_{n}\right)$ may in fact be larger than the expectation lower bound only by a constant factor. Wormald [18] conjectures that this factor is not larger than 2.856. It is appropriate to mention here a related result of Cuckler [7, who proved that every regular tournament on $n$ vertices contains at least $n ! /(2+o(1))^{n}$ Hamilton cycles, thereby resolving a conjecture of Thomassen [15]. Recently, Ferber, Krivelevich and Sudakov [8] significantly generalized Cuckler's result showing that an analogous result holds when the host graph is not a tournament but any regular orientation 
with degree at least $c n$ where $c>3 / 8$, namely, there are $((c+o(1)) n / e)^{n}$ Hamilton cycles. Finally, we refer the interested reader to an excellent survey of Kuhn and Osthus [12] on Hamilton cycles in directed graphs.

The moral of the results of [1] and [18] is that $T(H)$ and even $R(H)$ "beat" the expectation lower bound by a constant factor for $H=C_{n}$ and $H=P_{n}$. Hence, it is of interest to determine which other orientations $H$ have the property that $T(H)$ and $R(H)$ are larger than $n ! / 2^{e(H)}$ by a constant factor. Clearly, not every $H$ has this property. For let $M_{t}$ be the orientation of a perfect matching of $t$ edges. It is straightforward to verify that $G\left(M_{n / 2}\right)=n ! / 2^{n / 2}$ for every tournament $G$ with $n$ vertices. It seems that the regularity of $C_{n}$ and the almost regularity of $P_{n}$ is what makes them prone to appear more than the expected number of times in some carefully designed (regular) tournaments. Our main result in fact proves this fact in a very general setting which we next define. As it turns out, this setting is general enough to include all Eulerian orientations of bounded maximum degree as well as all "nontrivial" balanced orientations of bounded maximum degree.

For an orientation $H$, let plus $(H)$ be the number of directed paths of length 2 in $H$ and let $\operatorname{minus}(H)$ be the number of anti-directed paths of length 2 . Namely,

$$
\operatorname{plus}(H)=\sum_{v \in V(H)} d^{+}(v) d^{-}(v), \operatorname{minus}(H)=\sum_{v \in V(H)}\left(\begin{array}{c}
d^{+}(v) \\
2
\end{array}\right)+\left(\begin{array}{c}
d^{-}(v) \\
2
\end{array}\right) .
$$

For an orientation $H$, recall that $\hat{H}$ denotes the underlying graph of $H$. We define the maximum degree of $H$ as $\Delta(H)=\Delta(\hat{H})$. For $\epsilon>0$ and a positive integer $k$ we say that $H$ is an $(\epsilon, k)$ consistent orientation if $H$ has $n$ vertices, $\delta(H) \leq k$ and $p l u s(H)-\operatorname{minus}(H) \geq \epsilon n$. The following is our main result.

Theorem 1 Let $\epsilon>0$ and let $k$ be a positive integer. There exists $N=N(\epsilon, k)$ and $\delta=\delta(\epsilon, k)$ such that for every odd $n>N$ and every $(\epsilon, k)$-consistent orientation $H$ with $n$ vertices, $R(H) \geq$ $(1+\delta) n ! / 2^{e(H)}$.

When $n$ is odd, we trivially have $R(H) \leq T(H)$. When $n$ is even, $R(H)$ is not defined, but we can define it as the maximum number of labeled copies of $H$ in an $n$-vertex balanced tournament (i.e. tournaments with minimum in-degree $n / 2-1$ ). With this definition, $R(H) \leq T(H)$ regardless of the parity of $n$. The following can be proved as a corollary of our main result.

Corollary 1.1 Let $\epsilon>0$ and let $k$ be a positive integer. There exists $N=N(\epsilon, k)$ and $\delta=\delta(\epsilon, k)$ such that for every $n>N$ and every $(\epsilon, k)$-consistent orientation $H$ with $n$ vertices, $T(H) \geq$ $R(H) \geq(1+\delta) n ! / 2^{e(H)}$.

As mentioned earlier, the family of $(\epsilon, k)$-consistent orientations is quite large, and contains, for example, all Eulerian orientations of maximum degree $k$ using $\epsilon=1$ as well as all balanced orientations with maximum degree $k$ and not too many isolated vertices, using a sufficiently small $\epsilon$, as the following proposition shows. 
Proposition 1.2 Let $H$ be a balanced orientation with maximum degree $k$ and with at least $(0.5+$ $\epsilon) n$ edges. Then $H$ is a $(2 \epsilon / k, k)$-consistent orientation. Let $H$ be an Eulerian orientation with maximum degree $k$. Then $H$ is a $(1, k)$-consistent orientation.

We note that we need a balanced orientation to have more than 0.5 edges in order to apply Theorem 1 or Corollary 1.1 due to the above example which shows that $M_{n / 2}$, which is a balanced orientation with $n / 2$ edges, has $T\left(M_{n / 2}\right)=n ! / 2^{n / 2}$.

Interestingly, our proof can be optimized to yield relatively large values for $\delta$ when $H$ is an Eulerian orientation. This is best demonstrated in the case of $k$-regular orientations for which we have the following theorem.

Theorem 2 Let $H$ be a $k$-regular orientation with $n$ vertices. Then $R(H) \geq\left(e^{k}-o(1)\right) n ! / 2^{n k}$.

Notice that the case $k=1$ in Theorem 2 implies the theorem in [1. Let us outline in very high level the similarities and differences between the proof in [1] and the proof of Theorem 1, The idea in [1] is to fix some maximal packing of $K_{n}$ with edge-disjoint triangles (packing almost all edges), and then examine the probability space of random tournaments obtained by orienting each triangle as a directed triangle in one of the two possible directions. A Hamilton cycle (corresponding to a permutation) either intersects each triangle in one or two edges, and it is possible to estimate rather precisely, using Brun's Sieve, the probability that all its $n$ edges agree with the orientation of the random tournament. In our proof of Theorem 1 we also fix some maximal packing of $K_{n}$ into edge-disjoint cliques. However, we cannot take these cliques to be just triangles for the sheer reason that $\hat{H}$ many have many triangles and there is very high probability that in a random permutation, few of these triangles in $\hat{H}$ will actually identify with a triangle of the packing. As some (or even all) of these triangles might be $T_{3}$ 's in $H$, there is very high chance that the permutation will be inconsistent with every orientation of the random tournament. In fact, we will need to take our cliques in the packing to be $K_{t}$ 's where $t$ is very large, but still constant. But now, there are many more possibilities on how a random permutation might intersect each $K_{t}$ and using Brun's sieve to estimate the probability that all edges of $H$ are consistent with the random tournament becomes very difficult. Instead we use a different method based on the inequality of arithmetic and geometric means which reduces the computation to a feasible one.

In the following section we prove some basic lemmas which are needed for the proof of the main result, Theorem 1. Section 3 then contains the proof of Theorem 1, In Section 4 we consider balanced and Eulerian orientations and prove Proposition 1.2 and Theorem 2, The final section contains some concluding remarks and in particular, it is shown how to adjust Theorem 1 in order to obtain Corollary 1.1 . 


\section{Lemmata}

Let $G$ be a graph on vertex set $[n]$. A decomposition of $G$ is a family $\mathcal{D}$ of pairwise edge-disjoint labeled subgraphs of $G$ such that each edge of $G$ is covered by an element of $\mathcal{D}$. If all elements of $\mathcal{D}$ are isomorphic to the same graph $H$ then $\mathcal{D}$ is called an $H$-decomposition. The following is a special case of a seminal result of Wilson [17].

Lemma 2.1 Let $t \geq 3$ be an integer. There exists $N=N{ }_{2.1}(t)$ such that for all $n>N$, if $n \equiv 1, t \bmod t(t-1)$, then $K_{n}$ has a $K_{t}$-decomposition.

We note that the requirement that $n \equiv 1, t \bmod t(t-1)$ is a trivial necessary condition for the existence of a $K_{t}$-decomposition as clearly we must have that $t-1$ divides $n-1$ and that $\left(\begin{array}{l}t \\ 2\end{array}\right)$ divides $\left(\begin{array}{l}n \\ 2\end{array}\right)$. When $n$ is not of this form we cannot have a $K_{t}$-decomposition but we can still have a very large set of edge-disjoint $K_{t}$ 's. In fact, a result of Gustavsson [10] states that if $G$ is a graph on $n$ vertices such that $e(G)$ is divisible by $\left(\begin{array}{l}t \\ 2\end{array}\right)$ and that each vertex degree is divisible by $t-1$, then $G$ will have a $K_{t}$-decomposition if we assume that the minimum degree of $G$ is sufficiently large. Gustavsson's result also follows as a special case of a seminal result of Keevash on the existence of designs [1]. We need here the following even more special case.

Lemma 2.2 Let $t \geq 3$ be an integer and let $d \geq 1$. There exists $N=N \overline{2.2}(t, d)$ such that for all $n>N$ he following holds. Suppose $G$ is a graph with $n$ vertices, minimum degree at least $n-d$ and suppose furthermore that $e(G)$ is divisible by $\left(\begin{array}{l}t \\ 2\end{array}\right)$ and that each vertex degree is divisible by $t-1$. Then $G$ has a $K_{t}$-decomposition.

We note that the best known constants for $N=N_{2.2}(t, d)$ are rather huge. In fact, in our proof of Theorem 11 we could have settled for a weaker result than Lemma 2.2 which only guarantees that $K_{n}$ has a packing into edge-disjoint copies of $K_{t}$ which covers all but $o\left(n^{2}\right)$ edges of $K_{n}$. This weaker statement, which actually follows as a special case from a hypergraph packing result of Rödl [13], works with a much smaller $N$. However, using this weaker variant would have made the proof of Theorem 1 considerably more involved, due in part to the fact that we would still need to show that the leftover uncovered edges can be decomposed into constant size graphs.

Lemma 2.3 Let $t \geq 3$ be an odd integer. There exists $N=N[2.3(t)$ such that for all odd $n>N$ the following holds. There exists a graph $B$ on $n$ vertices such that $B$ has maximum degree at most $3 t-5$ and $B$ can be edge-decomposed into at most $n(t-3) / 6$ triangles, at most $t-3$ cycles of length 4 and at most $t-1$ copies of $K_{2 t-1}$. Furthermore, $K_{n}$ can be edge-decomposed into copies of $K_{t}$ and at most one copy of $B$.

Proof. Let $N \overline{2.3}(t)=N \overline{2.2}(t, 3 t-4)$. Suppose that $t \geq 3$ is odd and $n>N[2.3](t)$ is also odd. Let $q \equiv n \bmod t-1$ where $q \in\{1,3,5, \ldots, t-2\}$. 
Our first step is performed in $(q-1) / 2$ iterations (if $q=1$ the first step in ignored). In the first iteration, we remove from $K_{n}$ a set of pairwise vertex-disjoint triangles and at most two cycles of length four that cover each vertex precisely once. This is trivially doable as each number larger than 5 can be written as $3 x+4 y$ where $x$ and $y$ are nonnegative integers and $y \leq 2$. After the first iteration the remaining graph $G_{1}$ has $n$ vertices and each degree is $n-3$. In the second iteration (if there is one), we do the same, removing from $G_{1}$ a set of pairwise vertex-disjoint triangles and at most two cycles of length four that cover each vertex precisely once. This is again doable (less trivially now) using, say, a theorem of Corrádi and Hajnal [6] (with lots of room to spare as this theorem actually enable us do $\Theta(n)$ iterations, while we only need constantly many iterations). After the last iteration (iteration $(q-1) / 2$ ) the remaining graph $G^{\prime}$ has $n$ vertices and each degree is $n-q$. The graph of the deleted edges is, by construction, a regular graph $B^{\prime}$ with $n$ vertices and degree $q-1$, edge-decomposed into at most $n(q-1) / 6 \leq n(t-3) / 6$ triangles and at most $q-1 \leq t-3$ cycles of length 4 .

Notice now that each vertex degree of $G^{\prime}$ is divisible by $t-1$. However, the number of edges of $G^{\prime}$ is not necessarily divisible by $t(t-1) / 2$. Nevertheless, $e\left(G^{\prime}\right)$ is divisible by $(t-1) / 2$ since Indeed

$$
e\left(G^{\prime}\right)=\left(\begin{array}{l}
n \\
2
\end{array}\right)-e\left(B^{\prime}\right)=\left(\begin{array}{l}
n \\
2
\end{array}\right)-\frac{n(q-1)}{2}=\frac{n(n-q)}{2} \equiv 0 \bmod \frac{t-1}{2} .
$$

The second step further removes several pairwise vertex-disjoint copies of $K_{2 t-1}$ until the number of edges of the remaining graph is divisible by $t(t-1) / 2$. To show this is possible we first observe that since $t$ is odd,

$$
\operatorname{gcd}\left(e\left(K_{2 t-1}\right), \frac{t(t-1)}{2}\right)=\operatorname{gcd}\left((2 t-1)(t-1), \frac{t(t-1)}{2}\right)=\frac{t-1}{2} .
$$

So indeed after removing at most $t-1$ copies of $K_{2 t-1}$ the number of edges becomes divisible by $t(t-1) / 2$. We can remove these many copies vertex-disjointly since the degree of $G^{\prime}$ is $n-q$, so Turán's Theorem [16] lets us do this deletion greedily (with lots of room to spare). Finally, we arrive at the remaining graph $G$ which has $n$ vertices, its number of edges is divisible by $t(t-1) / 2$, each vertex degree of $G$ is either $n-q$ or $n-q-(2 t-2)$ so each vertex degree is divisible by $t-1$. Furthermore, the minimum degree of $G$ is at least $n-q-2 t+2 \geq n-3 t+4$. Thus, by Lemma 2.2. $G$ has a $K_{t}$-decomposition. The set of removed edges is a graph $B$ that is edge-decomposed into at most $n(t-3) / 6$ triangles, at most $t-3$ cycles of length 4 and at most $t-1$ copies of $K_{2 t-1}$. Furthermore, the maximum degree of $B$ is at most $(n-1)-\delta(G)=3 t-5$.

Definition 2.4 (adjusted $t$-decomposition) For an odd integer $t \geq 3$ and an odd integer $n$, we say that a decomposition $\mathcal{D}$ of $K_{n}$ is an adjusted $t$-decomposition if the set of edges of all elements of $\mathcal{D}$ that are not isomorphic to $K_{t}$ form a graph $B$ on $n$ vertices with maximum degree at most $3 t-5$. Furthermore, the elements of $\mathcal{D}$ that decompose $B$ are either at most $n(t-3) / 6$ triangles, at most $t-3$ cycles of length 4 and at most $t-1$ copies of $K_{2 t-1}$. 
Lemma 2.3 proves that for all odd $t \geq 3$ and all sufficiently large odd $n$, an adjusted $t$-decomposition exists.

Definition 2.5 (atypical copy, typical copy) Let $t \geq 3$ be an odd integer an let $\mathcal{D}$ be an adjusted $t$-decomposition of $K_{n}$ ( $n$ odd). Let $\mathcal{D}^{\prime}$ denote the subset of elements of $\mathcal{D}$ that are not copies of $K_{t}$. Let $H$ be an undirected graph with $n$ vertices. We say that a labeled copy of $H$ in $K_{n}$ is atypical if at least one of the following holds:

(i) some element of $\mathcal{D}^{\prime}$ contains two (or more) edges of $H$,

(ii) some $K_{t}$ of $\mathcal{D}$ contains three (or more) edges of $H$ where the three edges do not induce a triangle in $H$.

A labeled copy of $H$ which is not atypical is typical.

Observe that if $H$ is dense, then many of the labeled copies are atypical. For example, if $H=K_{n}$, then all $n$ ! labeled copies are atypical (unless $t=3$ and $\mathcal{D}$ is a Steiner triple system). The following lemma proves that if $H$ is sparse, then most labeled copies of $H$ will be typical.

Lemma 2.6 Let $t \geq 3$ be an odd integer an let $\mathcal{D}$ be an adjusted $t$-decomposition of $K_{n}$ ( $n$ odd). Let $H$ be an undirected graph with $n$ vertices and maximum degree $d$. Then there are at most $11 d^{3} t^{4}(n-1)$ ! atypical labeled copies of $H$ in $K_{n}$.

Proof. It is equivalent, although more convenient, to upper bound the probability that a random labeled copy of $H$ is atypical. So for $\sigma \in S_{n}$ drawn at random, we let $H_{\sigma}$ correspond to the random labeled copy of $H$. Let us first estimate the probability that condition (i) in Definition 2.5 holds. Let $\mathcal{D}^{\prime}$ denote the subset of elements of $\mathcal{D}$ that are not copies of $K_{t}$. Recall that the elements of $\mathcal{D}^{\prime}$ are either triangles, 4-cycles or copies of $K_{2 t-1}$. Fix some triangle $\{x, y, z\}$ of $\mathcal{D}^{\prime}$. What is the probability that $H_{\sigma}$ contains the edge $x y$ and also the edge $y z$ ? Given that the vertex of $H$ mapped to $y$ is, say, $u$, this occurs only if two neighbors of $u$ in $H$ map to $x$ and $z$. Since the maximum degree of $H$ is $d$, this occurs with probability at most $d(d-1) /((n-1)(n-2))$. Hence, the probability that $\{x, y, z\}$ contains two (or three) edges of $H_{\sigma}$ is at most $3 d(d-1) /((n-1)(n-2))$. Since $\mathcal{D}$ is an adjusted $t$-decomposition, $\mathcal{D}^{\prime}$ has at most $n(t-3) / 6$ triangles. So the probability that some triangle of $\mathcal{D}^{\prime}$ contains two or more edges of $H_{\sigma}$ is at most

$$
\frac{n(t-3)}{6} \cdot \frac{3 d(d-1)}{(n-1)(n-2)} .
$$

The overall number of edges in the 4-cycles of $\mathcal{D}^{\prime}$ is at most $4(t-3)$ and the overall number of edges in the $K_{2 t-1}$ copies of $\mathcal{D}^{\prime}$ is at most $(2 t-1)(t-1)^{2}$. The probability of any one of these $4(t-3)+(2 t-1)(t-1)^{2}$ edges to belong to $H_{\sigma}$ is $e(H) /\left(\begin{array}{l}n \\ 2\end{array}\right)$. Since $e(H) \leq n d / 2$, the probability that at least one of the edges of a 4 -cycle of $\mathcal{D}^{\prime}$ or a $K_{2 t-1}$ element of $\mathcal{D}^{\prime}$ will be in $H_{\sigma}$ is at most

$$
\left(4(t-3)+(2 t-1)(t-1)^{2}\right) \frac{d}{n-1} .
$$


Summing (11) and (2) we obtain that the probability that some element of $\mathcal{D}^{\prime}$ contains two (or more) edges of $H_{\sigma}$ is at most

$$
\frac{n(t-3)}{6} \cdot \frac{3 d(d-1)}{(n-1)(n-2)}+\left(4(t-3)+(2 t-1)(t-1)^{2}\right) \frac{d}{n-1}<\frac{8 t^{3} d^{2}}{n}
$$

We next compute the probability that some $K_{t}$ copy of $\mathcal{D}$ contains three or more edges of $H_{\sigma}$, where these three edges do not induce a triangle. Consider three edges $e, f, g$ of $H$ which do not form a triangle. Then they span either 4,5 or 6 vertices of $H$. Consider first the case that they span 6 vertices, so $e, f, g$ form a matching. Given that $e$ is placed in some $K_{t}$ element of $\mathcal{D}$, the probability that also $f$ and $g$ fall in this element is precisely the probability that their four endpoints are mapped to vertices of this element which is

$$
\frac{(t-2)(t-3)(t-4)(t-5)}{(n-2)(n-3)(n-4)(n-5)}<\frac{t^{4}}{n^{4}} .
$$

Since the number of choices for $e, f, g$ in $H$ is less than $e(H)^{3}<d^{3} n^{3}$, the probability that any three such edges fall in the same $K_{t}$ copy of $\mathcal{D}$ is at most

$$
d^{3} n^{3} \cdot \frac{t^{4}}{n^{4}} \leq \frac{d^{3} t^{4}}{n}
$$

Consider next the case that $e, f, g$ span 5 vertices. So, we must have that, say $e$ is disjoint from $f$ and $g$, and $f, g$ share an endpoint. Given that $e$ is in some $K_{t}$ element of $\mathcal{D}$, the probability that also $f$ and $g$ fall in this element is precisely the probability that their three endpoints are mapped to vertices of this element which is

$$
\frac{(t-2)(t-3)(t-4)}{(n-2)(n-3)(n-4)}<\frac{t^{3}}{n^{3}} .
$$

Since the number of choices for $e$ is $e(H)$ and the number of choices for $f, g$ sharing an endpoint is less than $n d^{2}$, the probability that any three such edges fall in the same $K_{t}$ copy of $\mathcal{D}$ is at most

$$
d^{3} n^{2} \cdot \frac{t^{3}}{n^{3}} \leq \frac{d^{3} t^{3}}{n} \text {. }
$$

Consider last the case that $e, f, g$ span 4 vertices (so either a path on three edges or a star on three edges). Given that $e$ is in some $K_{t}$ element of $\mathcal{D}$, the probability that also $f$ and $g$ fall in this element is precisely the probability that the two vertices of $f$ and $g$ that are not endpoints of $e$ are mapped to vertices of this element which is

$$
\frac{(t-2)(t-3)}{(n-2)(n-3)}<\frac{t^{2}}{n^{2}}
$$

Since the number of choices for stars with three edges in $H$ is less than $n d^{3} / 2$ and the number of choice of paths on three edges in $H$ is less than $e(H) d^{2} \leq n d^{3} / 2$, the probability that any three such edges fall in the same $K_{t}$ copy of $\mathcal{D}$ is at most

$$
d^{3} n \cdot \frac{t^{2}}{n^{2}} \leq \frac{d^{3} t^{2}}{n}
$$


Summing (3/45]6), we obtain that the probability that $H_{\sigma}$ is atypical is at most

$$
\frac{8 t^{3} d^{2}}{n}+\frac{d^{3} t^{4}}{n}+\frac{d^{3} t^{3}}{n}+\frac{d^{3} t^{2}}{n}<\frac{11 d^{3} t^{4}}{n} .
$$

Hence the number of atypical labeled copies of $H$ in $K_{n}$ is at most $11 d^{3} t^{4}(n-1)$ !.

Definition 2.7 (consistency, inconsistency, disjointness) A pair of edges of an orientation is called consistent if they share a common vertex and that vertex is the tail of one of them and the head of the other. A pair of edges of an orientation is inconsistent if they share a common vertex and that vertex is the tail of both of them or the head of both of them. A pair of edges of an orientation that is neither consistent nor inconsistent is disjoint (they do not share a common vertex).

Observe that $\operatorname{plus}(H)$ is the number of consistent pairs while minus $(H)$ is the number of inconsistent pairs.

Let $T$ be a tournament on vertex set $[t]$. For a permutation $\sigma \in S_{t}$ we let $T_{\sigma}$ denote the automorphism of $T$ obtained by the permutation $\sigma$ on the vertex set $[t]$.

Lemma 2.8 Let $t \geq 3$ be odd. Let $R$ be a regular tournament on vertex set $[t]$. Consider the symmetric probability space whose elements are all the $R_{\sigma}$ for $\sigma \in S_{t}$. Then, for any ordered triple $(x, y, z)$ of distinct vertices from $[t]$, the probability that the edge with endpoints $\{x, y\}$ is consistent with the edge with endpoints $\{y, z\}$ is $(t-1) /(2 t-4)$. Equivalently, the probability that this pair of edges is inconsistent is $(t-3) /(2 t-4)$. The probability that the triple $x, y, z$ induces a $C_{3}$ is $\frac{t+1}{4(t-2)}$. Equivalently, the probability that it induces a $T_{3}$ is $\frac{3(t-3)}{4(t-2)}$.

Proof. Suppose, without loss of generality that $(x, y)$ is an edge of $R_{\sigma}$. Since $R$ is a regular tournament, $y$ is a head of $(t-1) / 2$ other edges connecting $y$ to the remaining $t-2$ vertices. Thus, the probability that $(y, z) \in R_{\sigma}$ is $((t-1) / 2) /(t-2)=(t-1) /(2 t-4)$. The complementary event of being inconsistent has probability $1-(t-1) /(2 t-4)=(t-3) /(2 t-4)$. A regular tournament with $t$ vertices has exactly $t(t-1)(t-3) / 8$ copies of $T_{3}$. Hence the probability that $x, y, z$ induce a $T_{3}$ is $\frac{3(t-3)}{4(t-2)}$. The complementary event of being a $C_{3}$ has probability $\frac{t+1}{4(t-2)}$.

Finally, we need the following lemma.

Lemma 2.9 For all $\epsilon>0$, if $t$ is a sufficiently large integer, then

$$
\frac{t+1}{t-2} \geq\left(\frac{t-1}{t-2}\right)^{3-\epsilon}
$$

For all $K>0$, if $\rho>0$ is sufficiently small, then

$$
\left(1-\rho^{2}\right)^{K}(1+\rho) \geq 1+\frac{\rho}{2}
$$


Proof. The first inequality is equivalent to

$$
(t+1)(t-2)^{2} \geq(t-1)^{3}\left(\frac{t-2}{t-1}\right)^{\epsilon}
$$

which, in turn, is equivalent to

$$
1+\frac{1}{t-2} \geq\left(1+\frac{3 t-5}{t^{3}-3 t^{2}+4}\right)^{\frac{1}{\epsilon}}
$$

which clearly holds for all sufficiently large $t$ as a function of $\epsilon$. For the second claim, we may clearly assume that $K$ is an integer, and the left hand side is

$$
\left(1-\rho^{2}\right)^{K}(1+\rho)=1+\rho-K \rho^{2}-O\left(\rho^{3}\right)
$$

which is larger than $1+\rho / 2$ for all sufficiently small $\rho$ as a function of $K$.

\section{Proof of Theorem 1}

A pair of edges with a common endpoint is induced if the two non-common endpoints are not connected. For an orientation $H$, let $c(H)$ denote the number of induced consistent pairs of edges of $H$ and denote by $i(H)$ the number of induced inconsistent pairs of edges of $H$. Let $f(H)$ denote the number of directed triangles (copies of $C_{3}$ ) in $H$ and let $g(H)$ denote the number of acyclic triangles (copies of $T_{3}$ ) in $H$.

It will be more convenient to prove the following theorem which implies Theorem 1 .

Theorem 3 For any $\epsilon>0$ and any positive integer $k$, there exist $\delta=\delta(\epsilon, k)$ and $N=N(\epsilon, k)$ such that the following holds. Suppose $n>N$ is odd and $H$ is an orientation with $n$ vertices, $\Delta(H) \leq k$ and $c(H)+(3-\epsilon) f(H)-i(H)-g(H) \geq \epsilon n$. Then $R(H) \geq(1+\delta) n ! / 2^{e(H)}$.

Lemma 3.1 Theorem 3 implies Theorem 1 .

Proof. Let $H$ be an orientation. Note that each $C_{3}$ in $H$ consists of three directed paths of length 2, while each $T_{3}$ in $H$ consists of a single directed path of length 2 and two anti-directed paths of length 2. Thus,

$$
\operatorname{plus}(H)=3 f(H)+c(H)+g(H), \operatorname{minus}(H)=2 g(H)+i(H) .
$$

In particular plus $(H)-\operatorname{minus}(H)=c(H)+3 f(H)-i(H)-g(H)$. Let $\epsilon>0$ and $k$ be a positive integer. Let $\gamma=\epsilon /\left(k^{2}+1\right)$ and suppose that $n>N(\gamma, k)$ is odd where $N$ in the constant from Theorem 3. Now, suppose that $H$ is $(\epsilon, k)$-consistent with $n$ vertices. Thus, in particular, $\operatorname{plus}(H)-\operatorname{minus}(H) \geq \epsilon n$. Observe that since $\delta(H) \leq k$, then the number of $C_{3}$ in $G$ is smaller than $k^{2} n$, that is, $f(H) \leq k^{2} n$. Now,

$$
c(H)+(3-\gamma) f(H)-i(H)-g(H)=\operatorname{plus}(H)-\operatorname{minus}(H)-\gamma f(H) \geq \epsilon n-\gamma k^{2} n \geq \gamma n .
$$


Hence, by Theorem 3 with $\delta=\delta(\gamma, k)$, there are regular tournaments with $n$ vertices that contain at least $(1+\delta) n ! / 2^{e(H)}$ labeled copies of $H$.

Proof of Theorem 3 . Let $\rho=\frac{1}{t-2}$ where $t$ is the least positive integer satisfying the following three inequalities:

$$
\begin{aligned}
\frac{t+1}{t-2} & \geq\left(\frac{t-1}{t-2}\right)^{3-\epsilon} \\
\left(1-\rho^{2}\right)^{\frac{2 k^{2}}{\epsilon}}(1+\rho) & \geq 1+\frac{\rho}{2} \\
t & \geq \frac{2}{\epsilon} .
\end{aligned}
$$

By Lemma 2.9, $t$ is well-defined. Define $\delta=\rho / 4=\frac{1}{4 t-8} . N$ will be chosen sufficiently large as a function of $\epsilon, k, t$ (and thus, as a function of $\epsilon, k$ ) so that all claimed inequalities involving all $n>N$ hold throughout the proof.

Suppose that $n>N$ is odd and let $\mathcal{D}$ be an adjusted $t$-decomposition of $K_{n}$ which exists by Lemma 2.3 and our choice of constants. We consider the following probability space of regular tournaments on vertex set $[n]$. Let $R$ be any fixed regular tournament on the set of vertices $[t]$. Let $R^{*}$ be any fixed regular tournament on the set of vertices $[2 t-1]$. For each element $Z \in \mathcal{D}$ perform the following. If $Z$ is isomorphic to $K_{t}$, then orient it by placing a random copy of $R$ on it. More formally, suppose $Z$ consists of vertices $\left\{v_{1}, \ldots, v_{t}\right\}$. Take a random permutation $\sigma \in S_{t}$ and for each edge $v_{i} v_{j}$ orient it in the same direction of the edge of $R$ connecting $\sigma(i)$ and $\sigma(j)$. If $Z$ is isomorphic to $K_{2 t-1}$ (recall, there are only at most $t-1 \operatorname{such} Z$ in $\mathcal{D}$ ), then place a random copy of $R^{*}$ on it. If $Z$ is a 4-cycle, then orient the cycle as a directed cycle in one of the two possible directions, each direction equally likely. If $Z$ is a 3-cycle, then orient the cycle as a directed cycle in one of the two possible directions, each direction equally likely. This regularly orients each element of $\mathcal{D}$ (the $|\mathcal{D}|$ random orientations are independent of each other) and so the obtained random tournament is regular on vertex set $[n]$. Denote the obtained random regular tournament by $T$.

Let $\hat{H}$ denote the underlying undirected graph of $H$. Recall that there are $n$ ! labeled copies of $\hat{H}$ in $\hat{T}=K_{n}$ and we denote them by $\hat{H}_{\pi}$ for $\pi \in S_{n}$. Each $\hat{H}_{\pi}$ corresponds to a labeling $H_{\pi}$ of $H$. However, while $\hat{H}_{\pi}$ is trivially a labeled copy of $\hat{H}$ in $K_{n}, H_{\pi}$ is not necessarily a labeled copy of $H$ in $T$, since the direction of the edges may not agree with the direction of the edges in $T$. Let $\mathcal{L}$ denote the typical labeled copies of $\hat{H}$ in $K_{n}$ with respect to $\mathcal{D}$ (recall Definition 2.5). By Lemma 2.6 we know that $|\mathcal{L}| \geq n !-11 k^{3} t^{4}(n-1)$ !. We will prove that the expected number of typical labeled copies of $H$ in $T$ is already $(1+\delta) n ! / 2^{e(H)}$. In particular, this implies that there exists a regular $n$-vertex tournament for which the number of labeled copies of $H$ in it is at least $(1+\delta) n ! / 2^{e(H)}$.

For a labeled copy $\hat{H}_{\pi}$, let $C_{\pi}$ denote the number of induced consistent pairs of edges of $H_{\pi}$ that fall into the same element of $\mathcal{D}$, let $I_{\pi}$ denote the number of induced inconsistent pairs of edges of $H_{\pi}$ that fall into the same element of $\mathcal{D}$, let $F_{\pi}$ denote the number of $C_{3}$ of $H_{\pi}$ that fall into 
the same element of $\mathcal{D}$, and let $G_{\pi}$ denote denote the number of $T_{3}$ of $H_{\pi}$ that fall into the same element of $\mathcal{D}$. Trivially, $C_{\pi} \leq c(H), I_{\pi} \leq i(H), F_{\pi} \leq f(H)$ and $G_{\pi} \leq g(H)$ but they will usually be much smaller.

Now, if $\hat{H}_{\pi}$ is typical (namely, $\hat{H}_{\pi} \in \mathcal{L}$ ), observe that if two induced consistent or inconsistent pairs of edges fall into the same element of $\mathcal{D}$, then this element must be a $K_{t}$. Similarly, if the edges of a copy of $C_{3}$ or the edges of a copy of $T_{3}$ (namely, the edges of a triangle of $\hat{H}_{\pi}$ ) fall into the same element of $\mathcal{D}$, then this element must be a $K_{t}$. Let $X_{\pi}$ be the indicator random variable which equals 1 if $H_{\pi}$ is a labeled copy of $H$ in $T$ (recall: the random object here is $T$ ). Notice first an easy case: if no two edges of $H_{\pi}$ are in the same element of $\mathcal{D}$, then trivially $\operatorname{Pr}\left[X_{\pi}=1\right]=2^{-e(H)}$, since recall that each element of $\mathcal{D}$ is oriented independently, and any single edge has equal probability $\frac{1}{2}$ in both directions. The following claim determines $\operatorname{Pr}\left[X_{\pi}=1\right]$ for any typical copy $\hat{H}_{\pi} \in \mathcal{L}$ in terms of $C_{\pi}, I_{\pi}, F_{\pi}, G_{\pi}$.

Claim 3.2 Suppose $\hat{H}_{\pi} \in \mathcal{L}$, then

$\operatorname{Pr}\left[X_{\pi}=1\right]=\left(\frac{t-1}{4(t-2)}\right)^{C_{\pi}}\left(\frac{t-3}{4(t-2)}\right)^{I_{\pi}}\left(\frac{t+1}{8(t-2)}\right)^{F_{\pi}}\left(\frac{t-3}{8(t-2)}\right)^{G_{\pi}}\left(\frac{1}{2}\right)^{e(H)-2 C_{\pi}-2 I_{\pi}-3 F_{\pi}-3 G_{\pi}}$.

Proof. We say that an element of $\mathcal{D}$ is $\operatorname{good}$ (with respect to $H_{\pi}$ and $T$ ) if it does not contain edges of $H_{\pi}$, or if all the edges it contains from $H_{\pi}$ agree with $T$ (have the same orientation as in $T$ ). If an element of $\mathcal{D}$ contains a single edge of $H_{\pi}$, then it is good with probability $\frac{1}{2}$ since recall that in $T$ each edge direction is equally likely. Since $\hat{H}_{\pi}$ is typical, no element of $\mathcal{D}$ contains three edges of $H_{\pi}$ unless these three edges are a triangle of $\hat{H}_{\pi}$. So, we now consider an element of $\mathcal{D}$ that contains precisely two edges of $\hat{H}_{\pi}$, or a triangle of $\hat{H}_{\pi}$. Again, by typicality, this element is a $K_{t}$.

Assume first that it contains precisely two edges and these two edges do not share a common endpoint. Since $K_{t}$ was oriented in $T$ by taking a random permutation of $R$ (which is a regular tournament with $t$ vertices), the probability that both edges agree with $T$ is $(1 / 2) \cdot(1 / 2)=1 / 4$, so the probability that this element of $\mathcal{D}$ is good is $1 / 4$. Assume next that these two edges share a common endpoint. There are two cases here. Either these two edges in $H_{\pi}$ induce a consistent pair in $H_{\pi}$, or they induce an inconsistent pair in $H_{\pi}$. If they are consistent, say they are $(x, y)$ and $(y, z)$, then the probability that $(x, y)$ agrees with $T$ is $\frac{1}{2}$, and given that it agrees with $T$, the probability that also $(y, z)$ agrees with $T$ is, by Lemma 2.8, $(t-1) /(2 t-4)$. Thus, the probability that this element of $\mathcal{D}$ is good is $\frac{t-1}{4(t-2)}$. If they are inconsistent, say they are $(x, y)$ and $(z, y)$, then the probability that $(x, y)$ agrees with $T$ is $\frac{1}{2}$, and given that it agrees with $T$, the probability that also $(z, y)$ agrees with $T$ is, by Lemma $2.8,(t-3) /(2 t-4)$. Thus, the probability that this element of $\mathcal{D}$ is good is $\frac{t-3}{4(t-2)}$.

Assume next that it contains three edges which form a triangle in $\hat{H}_{\pi}$. Then it is either a $C_{3}$ in $H_{\pi}$ or a $T_{3}$ in $H_{\pi}$. Since $K_{t}$ was oriented in $T$ by taking a random permutation of $R$, the 
probability that the edges of a $C_{3}$ of $H_{\pi}$ falling in the same $K_{t}$ copy all agree with $T$ is, by Lemma 2.8, precisely $(t+1) /(8(t-2))$ since by Lemma 2.8, the three vertices are a directed triangle with probability $(t+1) /(4(t-2))$, and the two possible directions of the triangle are equally likely. Similarly, the probability that the edges of a $T_{3}$ of $H_{\pi}$ falling in the same $K_{t}$ copy all agree with $T$ is, by Lemma 2.8, precisely $(t-3) /(8(t-2))$ since by Lemma 2.8 , the three vertices are a $T_{3}$ with probability $3(t-3) /(4(t-2))$, and the six possible orientations of that $T_{3}$ are equally likely. Hence, the probability that the element of $\mathcal{D}$ is good in the $C_{3}$ case is $(t+1) /(8(t-2))$ and is $\frac{t-3}{8(t-2)}$ in the $T_{3}$ case.

Since the goodness of each element of $\mathcal{D}$ is independent of the goodness of any other element, and since there are $C_{\pi}$ elements that contain induced consistent pairs, $I_{\pi}$ elements that contain induced inconsistent pairs, $F_{\pi}$ elements that contain $C_{3}$ 's and $G_{\pi}$ elements that contains $T_{3}$ 's, we obtain the expression in the statement of the claim for the probability that all elements of $\mathcal{D}$ are good.

It follows from Claim 3.2 that the expected number of typical labeled copies of $H$ in $T$ is at least

$$
\sum_{\hat{H}_{\pi} \in \mathcal{L}}\left(\frac{t-1}{4(t-2)}\right)^{C_{\pi}}\left(\frac{t-3}{4(t-2)}\right)^{I_{\pi}}\left(\frac{t+1}{8(t-2)}\right)^{F_{\pi}}\left(\frac{t-3}{8(t-2)}\right)^{G_{\pi}}\left(\frac{1}{2}\right)^{e(H)-2 C_{\pi}-2 I_{\pi}-3 F_{\pi}-3 G_{\pi}}
$$

It hence remains to show that (8) is at least $(1+\delta) n ! / 2^{e(H)}$.

Let $C_{\text {avg }}$ denote the average of $C_{\pi}$ ranging over all $\hat{H}_{\pi} \in \mathcal{L}$ and let $I_{\text {avg }}$ denote the average of $I_{\pi}$ ranging over all $\hat{H}_{\pi} \in \mathcal{L}$. Similarly define $F_{\text {avg }}$ and $G_{\text {avg }}$. Let $C_{\text {avg }}^{*}$ denote the average of $C_{\pi}$ ranging over all $n$ ! labeled copies $\hat{H}_{\pi}$ and let $I_{\text {avg }}^{*}$ denote the average of $I_{\pi}$ ranging over all $n$ ! labeled copies $\hat{H}_{\pi}$ (so the difference between $C_{\text {avg }}^{*}$ and $C_{\text {avg }}$ is that the former averages over all labeled copies while the latter averages only over typical labeled copies and similarly for $I_{a v g}^{*}$ and $\left.I_{a v g}\right)$. Similarly define $F_{a v g}^{*}$ and $G_{a v g}^{*}$.

Let us first use $C_{\text {avg }}, I_{\text {avg }}, F_{\text {avg }}, G_{\text {avg }}$ to obtain a lower bound for (8).

\section{Claim 3.3}

$$
\begin{gathered}
\sum_{\hat{H}_{\pi} \in \mathcal{L}}\left(\frac{t-1}{4(t-2)}\right)^{C_{\pi}}\left(\frac{t-3}{4(t-2)}\right)^{I_{\pi}}\left(\frac{t+1}{8(t-2)}\right)^{F_{\pi}}\left(\frac{t-3}{8(t-2)}\right)^{G_{\pi}}\left(\frac{1}{2}\right)^{e(H)-2 C_{\pi}-2 I_{\pi}-3 F_{\pi}-3 G_{\pi}} \\
\geq|\mathcal{L}| \cdot\left(\frac{t-1}{4(t-2)}\right)^{C_{\text {avg }}}\left(\frac{t-3}{4(t-2)}\right)^{I_{\text {avg }}}\left(\frac{t+1}{8(t-2)}\right)^{F_{\text {avg }}}\left(\frac{t-3}{8(t-2)}\right)^{G_{\text {avg }}}\left(\frac{1}{2}\right)^{e(H)-2 C_{\text {avg }}-2 I_{\text {avg }}-3 F_{\text {avg }}-3 G_{\text {avg }}} .
\end{gathered}
$$

Proof. The inequality follows immediately from the inequality of arithmetic and geometric means.

So, our remaining task is to lower bound (9), i.e. the right hand side of the inequality in Claim 3.3. We will do so gradually by first proving good estimates for $C_{\text {avg }}^{*}, I_{\text {avg }}^{*}, F_{\text {avg }}^{*}, G_{\text {avg }}^{*}$ and then proving that $C_{a v g}$ is very close to $C_{a v g}^{*}$ and $I_{a v g}$ is very close to $I_{a v g}^{*}$ and respectively for $F_{a v g}$ and $G_{a v g}$. 
Let us consider first a case where $C_{\text {avg }}^{*}, I_{\text {avg }}^{*}, F_{\text {avg }}^{*}, G_{\text {avg }}^{*}$ are straightforward to compute. This is the case where all elements of $\mathcal{D}$ are isomorphic to $K_{t}$ (namely when the adjusted $t$-decomposition is actually a $K_{t}$-decomposition). Instead of counting the labeled copies is which two edges (or three edges of a triangle) appear in the same element of $\mathcal{D}$, it will be more convenient (though equivalent) to consider the labeled copies as a symmetric probability space of size $n$ ! where each labeled copy is equally likely. Consider a pair of edges of $H$ with a common endpoint (consistent, inconsistent, or inducing a triangle). Say the endpoints of the first edge are $u, v$ and the endpoints of the second edge is $v, w$. The probability that a randomly chosen labeled copy of $\hat{H}$ will place $u, v, w$ all in the same copy of $\mathcal{D}$ is precisely $(t-2) /(n-2)$. Since there are $c(H)$ consistent pairs in $H$, we have by linearity of expectation that $C_{\text {avg }}^{*}=c(H)(t-2) /(n-2)$ and similarly $I_{\text {avg }}^{*}=i(H)(t-2) /(n-2), F_{\text {avg }}^{*}=f(H)(t-2) /(n-2), G_{\text {avg }}^{*}=g(H)(t-2) /(n-2)$. The following claim estimates $C_{\text {avg }}^{*}, I_{\text {avg }}^{*}, F_{\text {avg }}^{*}, G_{\text {avg }}^{*}$ in the case that $\mathcal{D}$ is an arbitrary adjusted $t$-decomposition.

Claim 3.4 For a pair of edges of $\hat{H}$ with a common endpoint, let $p$ denote the probability that a labeled copy of $\hat{H}$ chosen at random will place both edges in the same element of $\mathcal{D}$. Then,

$$
\left|p-\frac{t-2}{n-2}\right| \leq \frac{3 t^{2}}{n^{2}}
$$

Proof. Let $\mathcal{D}^{\prime}$ be the subset of elements of $\mathcal{D}$ that are not isomorphic to $K_{t}$. By the definition of adjusted $t$-decomposition, all elements of $\mathcal{D}^{\prime}$ together contain at most $n(t-3) / 2+4(t-3)+(t-$ $1)^{2}(2 t-1)<n t$ edges.

Consider a pair of edges $e, f$ of $\hat{H}$ with a common endpoint. Say the endpoints of $e$ are $u, v$ and the endpoints of $f$ are $v, w$. Let $p$ denote the probability that a random labeled copy of $\hat{H}$ will place both $e$ and $f$ in the same element of $\mathcal{D}$. Let $p_{1}$ denote the probability that $e$ is placed in an element of $\mathcal{D}^{\prime}$. Let $q_{1}$ denote the probability that $f$ is placed in the same element as $e$ given that $e$ is placed in an element of $\mathcal{D}^{\prime}$. Let $q_{2}$ denote the probability that $f$ is placed in the same element as $e$ given that $e$ is placed in a $K_{t}$ element of $\mathcal{D}$. Thus, $p=p_{1} q_{1}+\left(1-p_{1}\right) q_{2}$.

Since all elements of $\mathcal{D}^{\prime}$ together contain at most $n t$ edges, we have that $p_{1} \leq n t /\left(\begin{array}{l}n \\ 2\end{array}\right)=2 t /(n-1)$. Since each element of $\mathcal{D}^{\prime}$ is either a triangle or a cycle of length 4 or a $K_{2 t-1}$, the probability that vertex $w$ (and hence $f$ ) is also placed in the same element of $\mathcal{D}^{\prime}$ in which $e$ is placed is at most $(2 t-3) /(n-2)$. Hence, $q_{1} \leq(2 t-3) /(n-2)$. Given that $e$ is placed in a $K_{t}$-element, the probability that vertex $w$ (and hence $f$ ) is also placed in that element is $q_{2}=(t-2) /(n-2)$. Hence,

$$
\left|p-\frac{t-2}{n-2}\right|=\left|p-q_{2}\right|=p_{1}\left|q_{1}-q_{2}\right| \leq \frac{2 t}{n-1} \cdot \frac{t-1}{n-2} \leq \frac{3 t^{2}}{n^{2}} .
$$

Now, since $C_{\text {avg }}^{*}=c(H) p, I_{\text {avg }}^{*}=i(H) p, F_{\text {avg }}^{*}=f(H) p, G_{\text {avg }}^{*}=g(H) p$ where $p$, as in the last claim, is the probability that two edges sharing an endpoint are placed in the same element of $\mathcal{D}$, we immediately obtain the following. 


\section{Corollary 3.5}

$$
\begin{gathered}
\left|C_{\text {avg }}^{*}-c(H) \frac{t-2}{n-2}\right| \leq c(H) \frac{3 t^{2}}{n^{2}}, \quad\left|I_{\text {avg }}^{*}-i(H) \frac{t-2}{n-2}\right| \leq i(H) \frac{3 t^{2}}{n^{2}} \\
\left|F_{\text {avg }}^{*}-f(H) \frac{t-2}{n-2}\right| \leq f(H) \frac{3 t^{2}}{n^{2}},\left|G_{\text {avg }}^{*}-g(H) \frac{t-2}{n-2}\right| \leq g(H) \frac{3 t^{2}}{n^{2}}
\end{gathered}
$$

We next show that $C_{\text {avg }}^{*}$ and $C_{a v g}$ are not far apart and similarly for $\left(I_{\text {avg }}^{*}, I_{\text {avg }}\right),\left(F_{\text {avg }}^{*}, F_{\text {avg }}\right)$, $\left(G_{\text {avg }}^{*}, G_{\text {avg }}\right)$.

\section{Claim 3.6}

$$
\begin{aligned}
& c(H)\left(\frac{t-2}{n-2}-\frac{3 t^{2}}{n^{2}}\right)-\frac{133 k^{3} t^{4} d^{2}}{\sqrt{n}} \leq C_{\text {avg }} \leq c(H)\left(\frac{t-2}{n-2}+\frac{23 k^{3} t^{5}}{n^{2}}\right) . \\
& i(H)\left(\frac{t-2}{n-2}-\frac{3 t^{2}}{n^{2}}\right)-\frac{133 k^{3} t^{4} d^{2}}{\sqrt{n}} \leq I_{\text {avg }} \leq i(H)\left(\frac{t-2}{n-2}+\frac{23 k^{3} t^{5}}{n^{2}}\right) . \\
& f(H)\left(\frac{t-2}{n-2}-\frac{3 t^{2}}{n^{2}}\right)-\frac{133 k^{3} t^{4} d^{2}}{\sqrt{n}} \leq F_{\text {avg }} \leq f(H)\left(\frac{t-2}{n-2}+\frac{23 k^{3} t^{5}}{n^{2}}\right) . \\
& g(H)\left(\frac{t-2}{n-2}-\frac{3 t^{2}}{n^{2}}\right)-\frac{133 k^{3} t^{4} d^{2}}{\sqrt{n}} \leq G_{\text {avg }} \leq g(H)\left(\frac{t-2}{n-2}+\frac{23 k^{3} t^{5}}{n^{2}}\right) .
\end{aligned}
$$

Proof. We prove for $C_{a v g}$. The proof for the others is identical. Let $C(H)$ be the set of triplets of vertices of $H$ that induce a consistent pair in $H$, thus $|C(H)|=c(H)$. A subset $S \subset C(H)$ is independent if any two elements of $S$ are disjoint (so their union is 6 vertices). For an independent subset $S \subset C(H)$ we say that a labeled copy of $\hat{H}$ is $S$-bad if for each triplet in $S$, all three vertices of the triplet appear in the same element of $\mathcal{D}$. Our goal is to prove that if $|S|$ is large, the probability of being $S$-bad is very small.

Fix some $S \subset C(H)$ with $s=|S|$ and suppose that $S=\left\{\left\{x_{i}, y_{i}, z_{i}\right\} \mid i=1, \ldots, s\right\}$. What is the probability that a random labeled copy of $\hat{H}$ is $S$-bad? Recall that each element of $\mathcal{D}$ is either a $K_{t}$, a $C_{3}$, a $C_{4}$ or a $K_{2 t-1}$. Consider first the triplet $\left\{x_{1}, y_{1}, z_{1}\right\}$. The probability that all three fall in the same element of $\mathcal{D}$ is at most $(2 t-3) /(n-2)$ (it is actually smaller since most elements of $\mathcal{D}$ are $K_{t}$ so the probability is very close to $(t-2) /(n-2)$ but we don't want to bother with a case analysis). Given that $\left\{x_{i}, y_{i}, z_{i}\right\}$ each fall in the same element of $\mathcal{D}$ for $i=1, \ldots, r-1$ (distinct triplets may or may not fall in the same element of $\mathcal{D}$ ) what is the probability that also $\left\{x_{r}, y_{r}, z_{r}\right\}$ fall in the same element of $\mathcal{D}$ ? Suppose we are given the information to which element of $\mathcal{D}$ each of the $3 r-1$ vertices of $x_{1}, y_{1}, z_{1}, x_{2}, y_{2}, z_{2} \ldots, x_{r-1}, y_{r-1}, z_{r-1}, x_{r}, y_{r}$ fall. The probability that $z_{r}$ also falls in the copy to which $x_{r}$ and $y_{r}$ fell is thus at most $(2 t-3) /(n-3 r+1)$. Hence, the probability that a random labeled copy of $\hat{H}$ is $S$-bad is at most

$$
\left(\frac{2 t-3}{n-3 s+1}\right)^{s}
$$


Recall that $\Delta(H) \leq k$ and therefore, trivially $c(H)<k^{2} n$ (and the same trivial upper bound holds for $i(H), f(H), g(H))$. Let us use, say, $s=\lfloor\sqrt{n}\rfloor$ for the remainder of the proof and say that a labeled copy of $\hat{H}$ is bad if it is $S$-bad for some independent $S \subset C(H)$ with $|S|=s=\lfloor\sqrt{n}\rfloor$. Thus, the probability that a random labeled copy of $\hat{H}$ is bad is at most

$$
\left(\begin{array}{c}
c(H) \\
s
\end{array}\right)\left(\frac{2 t-3}{n-3 s+1}\right)^{s} \leq\left(\frac{e k^{2} n}{s}\right)^{s}\left(\frac{2 t-3}{n-3 s+1}\right)^{s} \ll \frac{1}{n^{2}}
$$

where we have only used here that $n$ is sufficiently large as a function of $t$ and $k$. In other words, we have proved hat the number of bad labeled copies of $\hat{H}$ is at most $n ! / n^{2} \leq(n-2)$ !.

We will say that a labeled copy of $\hat{H}$ is very bad if at least $6 k^{2} s$ elements of $C(H)$ each appear together in the same element of $\mathcal{D}$. So the difference between being bad and very bad is that in the latter we don't insist that the triplets are independent. We claim that if a labeled copy is very bad, then it is also bad. Indeed, consider the graph $W$ whose vertices are the elements of $C(H)$, and two vertices of $W$ are connected if they are not disjoint (i.e. they span together less than 6 vertices). Since $\Delta(H) \leq k$, each vertex of $H$ appears in less than $2 k^{2}$ triplets. Hence, the maximum degree of $W$ is less than $6 k^{2}$. It follows that any set of $6 k^{2} s$ elements of $C(H)$ has an independent set of size at least $s$, hence if a labeled copy is very bad, then it is also bad, and thus, by (10), there are at most $(n-2)$ ! very bad labeled copies of $\hat{H}$.

We are now ready to compute our upper and lower bounds for $C_{a v g}$. Recall that by their definitions,

$$
\sum_{\pi \in S_{n}} C_{\pi}=n ! C_{\text {avg }}^{*}, \sum_{\hat{H}_{\pi} \in \mathcal{L}} C_{\pi}=|\mathcal{L}| C_{\text {avg }} .
$$

The upper bound follows rather simply from Lemma 2.6 which asserts that $|\mathcal{L}| \geq n !-11 k^{3} t^{4}(n-1)$ !. Indeed,

$$
C_{a v g}|\mathcal{L}|=n ! C_{a v g}^{*}-\sum_{\hat{H}_{\pi} \notin \mathcal{L}} C_{\pi} \leq n ! C_{a v g}^{*} .
$$


So,

$$
\begin{aligned}
C_{a v g} & \leq \frac{n ! C_{\text {avg }}^{*}}{|L|} \\
& =C_{\text {avg }}^{*}+\frac{(n !-|\mathcal{L}|) C_{\text {avg }}^{*}}{|\mathcal{L}|} \\
& \leq C_{\text {avg }}^{*}+\frac{11 k^{3} t^{4}(n-1) ! C_{a v g}^{*}}{|\mathcal{L}|} \\
& \leq C_{\text {avg }}^{*}+\frac{22 k^{3} t^{4} C_{\text {avg }}^{*}}{n} \\
& =C_{\text {avg }}^{*}\left(1+\frac{22 k^{3} t^{4}}{n}\right) \\
& \leq c(H)\left(\frac{t-2}{n-2}+\frac{3 t^{2}}{n^{2}}\right)\left(1+\frac{22 k^{3} t^{4}}{n}\right) \\
& \leq c(H)\left(\frac{t-2}{n-2}+\frac{23 k^{3} t^{5}}{n^{2}}\right) .
\end{aligned}
$$

Notice that we have used Claim 3.5 which asserts that $C_{\text {avg }}^{*} \leq c(H)\left(\frac{t-2}{n-2}+\frac{3 t^{2}}{n^{2}}\right)$.

For the lower bound, we need first to bound $\sum_{\hat{H}_{\pi} \notin \mathcal{L}} C_{\pi}$. We know that the number of $\hat{H}_{\pi} \notin \mathcal{L}$ is at most $11 k^{3} t^{4}(n-1)$ !. By the first part of the proof, at most $(n-2)$ ! of them are very bad, and for them we will use the trivial bound $C_{\pi} \leq c(H) \leq k^{2} n$. The others are not very bad, so for them we have $C_{\pi} \leq 6 k^{2} \sqrt{n}$. Thus,

$$
\sum_{\hat{H}_{\pi} \notin \mathcal{L}} C_{\pi} \leq 11 k^{3} t^{4}(n-1) ! \cdot 6 k^{2} \sqrt{n}+(n-2) ! k^{2} n .
$$

Now,

$$
\begin{aligned}
C_{a v g} & =\frac{n ! C_{\text {avg }}^{*}-\sum_{\hat{H}_{\pi} \notin \mathcal{L}} C_{\pi}}{|\mathcal{L}|} \\
& \geq C_{\text {avg }}^{*}-\frac{\sum_{\hat{H}_{\pi} \notin \mathcal{L}} C_{\pi}}{|\mathcal{L}|} \\
& \geq C_{\text {avg }}^{*}-\frac{2 \sum_{\hat{H}_{\pi} \notin \mathcal{L}} C_{\pi}}{n !} \\
& \geq C_{\text {avg }}^{*}-\frac{2 \cdot 11 k^{3} t^{4} \cdot 6 k^{2}}{\sqrt{n}}-\frac{2 k^{2}}{n-1} \\
& \geq C_{\text {avg }}^{*}-\frac{133 k^{5} t^{4}}{\sqrt{n}} \\
& \geq c(H)\left(\frac{t-2}{n-2}-\frac{3 t^{2}}{n^{2}}\right)-\frac{133 k^{5} t^{4}}{\sqrt{n}} .
\end{aligned}
$$

Notice that we have used Claim 3.5 which asserts that $C_{\text {avg }}^{*} \geq c(H)\left(\frac{t-2}{n-2}-\frac{3 t^{2}}{n^{2}}\right)$. 
Corollary 3.7 $C_{a v g}+(3-\epsilon) F_{a v g}-I_{a v g}-G_{a v g} \geq \frac{\epsilon}{2} t$.

Proof. By Claim 3.6.

$$
\begin{gathered}
C_{\text {avg }}+(3-\epsilon) F_{\text {avg }}-I_{\text {avg }}-G_{\text {avg }} \geq \\
(c(H)+(3-\epsilon) f(H))\left(\frac{t-2}{n-2}-\frac{3 t^{2}}{n^{2}}\right)-4 \frac{133 k^{5} t^{4}}{\sqrt{n}}-(i(H)+g(H))\left(\frac{t-2}{n-2}+\frac{23 k^{3} t^{5}}{n^{2}}\right) .
\end{gathered}
$$

By the assumption of Theorem 3, $c(H)+(3-\epsilon) f(H)-i(H)-g(H) \geq \epsilon n$. Also, trivially, each of $c(H), i(H), f(H), g(H)$ is at most $k^{2} n$ so the last inequality is at least

$$
\epsilon n \frac{t-2}{n-2}-O\left(\frac{1}{\sqrt{n}}\right) \geq \frac{\epsilon}{2} t
$$

for all $n$ sufficiently large as a function of $\epsilon, k, t$.

We are now ready to prove our final claim which completes the proof of Theorem 3 .

\section{Claim 3.8}

$$
\begin{gathered}
|\mathcal{L}| \cdot\left(\frac{t-1}{4(t-2)}\right)^{C_{\text {avg }}}\left(\frac{t-3}{4(t-2)}\right)^{I_{\text {avg }}}\left(\frac{t+1}{8(t-2)}\right)^{F_{\text {avg }}}\left(\frac{t-3}{8(t-2)}\right)^{G_{\text {avg }}}\left(\frac{1}{2}\right)^{e(H)-2 C_{\text {avg }}-2 I_{\text {avg }}-3 F_{\text {avg }}-3 G_{\text {avg }}} \\
\geq n !(1+\delta)\left(\frac{1}{2}\right)^{e(H)} .
\end{gathered}
$$

Proof. First observe that since $|\mathcal{L}| \geq n$ ! $-11 k^{3} t^{4}(n-1)$ !, then for all $n$ sufficiently large as a function of $\delta, t, k$ we have $|\mathcal{L}| \geq(1+\delta) n ! /(1+2 \delta)$. Hence it suffices to prove that

$$
\begin{gathered}
\left(\frac{t-1}{4(t-2)}\right)^{C_{\text {avg }}}\left(\frac{t-3}{4(t-2)}\right)^{I_{\text {avg }}}\left(\frac{t+1}{8(t-2)}\right)^{F_{\text {avg }}}\left(\frac{t-3}{8(t-2)}\right)^{G_{\text {avg }}}\left(\frac{1}{2}\right)^{e(H)-2 C_{\text {avg }}-2 I_{\text {avg }}-3 F_{\text {avg }}-3 G_{\text {avg }}} \\
\geq(1+2 \delta)\left(\frac{1}{2}\right)^{e(H)} .
\end{gathered}
$$

which is equivalent to proving that

$$
\left(\frac{t-1}{t-2}\right)^{C_{\text {avg }}}\left(\frac{t-3}{t-2}\right)^{I_{\text {avg }}}\left(\frac{t+1}{t-2}\right)^{F_{\text {avg }}}\left(\frac{t-3}{t-2}\right)^{G_{\text {avg }}} \geq 1+2 \delta .
$$

Recall that by our choice of $t$,

$$
\frac{t+1}{t-2} \geq\left(\frac{t-1}{t-2}\right)^{3-\epsilon}
$$

Hence it suffices to prove that

$$
\left(\frac{t-1}{t-2}\right)^{C_{a v g}+(3-\epsilon) F_{a v g}}\left(\frac{t-3}{t-2}\right)^{I_{a v g}+G_{a v g}} \geq 1+2 \delta .
$$


Now, by Claim 3.6 and the trivial bound $i(H)+g(H) \leq k^{2} n$ we have

$$
I_{\text {avg }}+G_{\text {avg }} \leq(i(H)+g(H))\left(\frac{t-2}{n-2}+\frac{23 k^{3} t^{5}}{n^{2}}\right) \leq(i(H)+g(H)) \frac{t-1}{n-2} \leq k^{2} t .
$$

Hence, by Corollary 3.7

$$
\begin{aligned}
& \left(\frac{t-1}{t-2}\right)^{C_{\text {avg }}+(3-\epsilon) F_{\text {avg }}}\left(\frac{t-3}{t-2}\right)^{I_{\text {avg }}+G_{a v g}} \\
= & \left(1-\frac{1}{(t-2)^{2}}\right)^{I_{\text {avg }}+G_{\text {avg }}}\left(\frac{t-1}{t-2}\right)^{C_{\text {avg }}+(3-\epsilon) F_{\text {avg }}-I_{\text {avg }}-G_{\text {avg }}} \\
\geq & \left(1-\frac{1}{(t-2)^{2}}\right)^{I_{\text {avg }}+G_{\text {avg }}}\left(\frac{t-1}{t-2}\right)^{\frac{\epsilon}{2} t} \\
\geq & \left(1-\frac{1}{(t-2)^{2}}\right)^{k^{2} t}\left(\frac{t-1}{t-2}\right)^{\frac{\epsilon}{2} t}
\end{aligned}
$$

So it remains to prove that

$$
\left(1-\frac{1}{(t-2)^{2}}\right)^{k^{2} t}\left(1+\frac{1}{t-2}\right)^{\frac{\epsilon}{2} t} \geq 1+2 \delta
$$

which is equivalent to proving

$$
\left(1-\frac{1}{(t-2)^{2}}\right)^{\frac{2 k^{2}}{\epsilon}}\left(1+\frac{1}{t-2}\right) \geq(1+2 \delta)^{\frac{2}{\epsilon t}}
$$

But recall that $\delta=\frac{1}{4(t-2)}$ and that $2 /(\epsilon t) \leq 1$ so the last inequality holds if the following inequality holds:

$$
\left(1-\frac{1}{(t-2)^{2}}\right)^{\frac{2 k^{2}}{\epsilon}}\left(1+\frac{1}{t-2}\right) \geq 1+\frac{1}{2 t-4}
$$

and indeed the last inequality holds by our choice of $t$.

Claim 3.8 proves the claimed lower bound for (9) and hence Theorem 3 holds. By Lemma 3.1 , Theorem 1 holds as well.

\section{Balanced and Eulerian orientations}

Proof of Proposition 1.2. Let $H$ be a balanced orientation with $n$ vertices, maximum degree $k$, and at least $(0.5+\epsilon) n$ edges. We first notice that the underlying graph $\hat{H}$ has at least $2 \epsilon n / k$

vertices with degree at least 2 . Indeed, otherwise the sum of the degrees of the vertices of $\hat{H}$ would have been less than $n+2 \epsilon n=(1+2 \epsilon) n$, contradicting the fact that is has at least $(0.5+\epsilon) n$ edges. 
Since $H$ is balanced, we have that for any vertex $v$ whose degree in $H$ is at least 2,

$$
d^{+}(v) d^{-}(v)-\left(\left(\begin{array}{c}
d^{+}(v) \\
2
\end{array}\right)+\left(\begin{array}{c}
d^{-}(v) \\
2
\end{array}\right)\right) \geq 1
$$

It follows that

$$
\operatorname{plus}(H)-\operatorname{minus}(H) \geq 2 \epsilon n / k
$$

which means that $H$ is a $(2 \epsilon / k, k)$-consistent orientation. If $H$ is an Eulerian orientation, then (12) holds for each $v \in V(H)$ so plus $(H)-\operatorname{minus}(H) \geq n$ which means that $H$ is a $(1, k)$-consistent orientation.

Proof of Theorem 2, We show that Theorem 2 follows from a careful optimization of the inequalities in the proof of Theorem 3. We first observe that if $H$ is a $k$-regular orientation, then $\operatorname{plus}(H)=k^{2} n$ while minus $(H)=k(k-1) n$ and recall that using the notation in Section 3 we have by (7) that $\operatorname{plus}(H)=3 f(H)+c(H)+g(H)=n k^{2}$ while $\operatorname{minus}(H)=2 g(H)+i(H)=k(k-1) n$.

Recalling Claim 3.3. our goal is to prove that for every $\gamma>0$, and for all sufficiently large $t$,

$|\mathcal{L}| \cdot\left(\frac{t-1}{4(t-2)}\right)^{C_{\text {avg }}}\left(\frac{t-3}{4(t-2)}\right)^{I_{\text {avg }}}\left(\frac{t+1}{8(t-2)}\right)^{F_{\text {avg }}}\left(\frac{t-3}{8(t-2)}\right)^{G_{\text {avg }}}\left(\frac{1}{2}\right)^{e(H)-2 C_{\text {avg }}-2 I_{\text {avg }}-3 F_{\text {avg }}-3 G_{\text {avg }}}$ is at least $\left(e^{k}-\gamma\right) n ! / 2^{n k}$ for all $n$ sufficiently large. As $e(H)=n k$ this is equivalent to proving that for every $\gamma>0$, and for all sufficiently large $t$,

$$
|\mathcal{L}|\left(\frac{t-1}{t-2}\right)^{C_{\text {avg }}}\left(\frac{t-3}{t-2}\right)^{I_{\text {avg }}}\left(\frac{t+1}{t-2}\right)^{F_{\text {avg }}}\left(\frac{t-3}{t-2}\right)^{G_{\text {avg }}} \geq\left(e^{k}-\gamma\right) n !
$$

holds for all $n$ sufficiently large.

By Lemma 2.6, $|\mathcal{L}| \geq n !-11 k^{3} t^{4}(n-1)$ ! so it is equivalent to prove that for all $t$ sufficiently large

$$
\left(\frac{t-1}{t-2}\right)^{C_{a v g}}\left(\frac{t-3}{t-2}\right)^{I_{a v g}}\left(\frac{t+1}{t-2}\right)^{F_{a v g}}\left(\frac{t-3}{t-2}\right)^{G_{a v g}} \geq e^{k}-\frac{\gamma}{2}
$$

holds for all $n$ sufficiently large.

Simple algebraic manipulation of the left hand side shows that it is equal to

$$
\left(\frac{t-1}{t-2}\right)^{C_{a v g}+3 F_{a v g}-I_{a v g}-G_{a v g}}\left(1-\frac{1}{(t-2)^{2}}\right)^{I_{a v g}+G_{a v g}}\left(1-\frac{3 t-5}{(t-1)^{3}}\right)^{F_{a v g}} .
$$

But now, from Claim 3.6 and the trivial bound $i(H)+g(H) \leq k^{2} n$ and $f(H) \leq k^{2} n$ we have as in (11) that

$$
I_{\text {avg }}+G_{\text {avg }} \leq k^{2} t \quad, \quad F_{\text {avg }} \leq k^{2} t
$$

Also, by Claim 3.6 .

$C_{a v g}+3 F_{a v g}-I_{a v g}-G_{a v g} \geq(c(H)+3 f(H))\left(\frac{t-2}{n-2}-\frac{3 t^{2}}{n^{2}}\right)-4 \frac{133 k^{5} t^{4}}{\sqrt{n}}-(i(H)+g(H))\left(\frac{t-2}{n-2}+\frac{23 k^{3} t^{5}}{n^{2}}\right)$. 
Now, since $c(H)+3 f(H)-i(H)-g(H)=k n$ and since each of $c(H), i(H), f(H), g(H)$ is at most $k^{2} n$ we have that

$$
C_{a v g}+3 F_{a v g}-I_{a v g}-G_{a v g} \geq k n \frac{t-2}{n-2}-O\left(\frac{1}{\sqrt{n}}\right) \geq k(t-3)
$$

for all $n$ sufficiently large as a function of $k, t$. It thus remains to show that

$$
\left(\frac{t-1}{t-2}\right)^{k(t-3)}\left(1-\frac{1}{(t-2)^{2}}\right)^{k^{2} t}\left(1-\frac{3 t-5}{(t-1)^{3}}\right)^{k^{2} t} \geq e^{k}-\frac{\gamma}{2}
$$

for all $t$ sufficiently large. This indeed holds as the first term in the left hand side tends to $e^{k}$ as $t \rightarrow \infty$ while the two other terms on the left hand side tend to 1 as $t \rightarrow \infty$.

It is easy to see that what really matters in the proof of Theorem 2 is not the $k$-regularity itself, but rather that $H$ has some bounded maximum degree and that $e(H)=n k$ and also plus $(H)-$ $\operatorname{minus}(H)=c(H)+3 f(H)-i(H)-g(H)=k n$. As for any even orientation (and hence any Eulerian orientation) we have $\operatorname{plus}(H)-\operatorname{minus}(H)=e(H)$ we obtain the following corollary.

Corollary 4.1 For any positive integer $K$ and any $\gamma>0$, there exists $N=N(K, \gamma)$ such that if $H$ is any Eulerian orientation with $n>N$ vertices, maximum degree at most $K$, and $k n$ edges, then $R(H) \geq\left(e^{k}-\gamma\right) n ! / 2^{n k}$.

\section{Concluding remarks}

Proof of Corollary 1.1. We slightly modify the definition of an adjusted $t$-decomposition to apply to the case where $n$ is even. So, assume the vertices of $K_{n}$ are labeled with $[n]$ where $n$ is even. Take an adjusted $t$-decomposition $\mathcal{D}^{*}$ on $[n-1]$ (note: we still assume that $t$ is odd). Now, to extend it to a decomposition of $K_{n}$ just add the following $n / 2$ elements $P_{1}, \ldots, P_{n / 2}$ where $P_{i}$ consists of the two edges $\{2 i-1, n\}$ and $\{2 i, n\}$ for $i=1, \ldots, n / 2-1$ while $P_{n / 2}$ is the single edge $\{n-1, n\}$. This decomposition $\mathcal{D}$ of $K_{n}$ is now defined as an adjusted $t$-decomposition. Notice that each element of $\mathcal{D}$ that is not in $\mathcal{D}^{*}$ is either a $K_{1,2}$ or a single edge. Now, in the proof of Theorem 3 where we randomly orient each element of $\mathcal{D}$, do as before for the elements of $\mathcal{D}^{*}$, while for each $P_{i}$ for $i=1, \ldots, n / 2-1$ orient it as a directed path of length 2 in one of the two possible directions. Also, orient the single edge $P_{n / 2}$ in one of the two possible direction. Notice that the obtained random orientation $T$ induces a regular tournament on $[n-1]$ and vertex $n$ has in-degree either $n / 2$ or $n / 2-1$, so $T$ is a balanced tournament. Now, all the rest of the proof of Theorem 3 carries over exactly in the the same way.

Recall that Wormald conjectured that $T\left(C_{n}\right)=\Theta\left(n ! / 2^{n}\right)$ and in fact that $T\left(C_{n}\right) \leq 2.856 n ! / 2^{n}$ for all sufficiently large $n$. It would thus be natural to conjecture that $T(H)=\Theta\left(n ! / 2^{e(H)}\right)$ for, say, all $(\epsilon, k)$-consistent orientations with $n$ vertices. 
Perhaps less ambitious, but possibly difficult, is to improve the constant $e^{k}$ in Theorem 2 and in Corollary 4.1. Recall that Wormald proved that for the Hamilton cycle (which applies the case $k=1$ ) the constant can be improved from $e$ to 2.855 . However, it seems difficult to try to generalize Wormald's argument to all, say, $k$-regular orientations as such orientations may have highly involved structure (they can contain, for example, arbitrary sub-tournaments on $\Theta(k)$ vertices) so it does not seem plausible to reduce the problem to a computer verification check of small constant size tournaments an in the proof in [18].

Finally, recall that the results of [2, 9] prove that $T\left(C_{n}\right)=O\left(n^{c} n ! / 2^{n}\right)$ for a relatively small constant $c$. It would be nice to prove an analogous result for say, $k$-regular orientations, stating $T(H)=O\left(n^{c} n ! / 2^{n k}\right)$ for some constant $c=c(k)$.

\section{References}

[1] I. Adler, N. Alon, and S. Ross. On the maximum number of Hamiltonian paths in tournaments. Random Structures and Algorithms, 18(3):291-296, 2001.

[2] N. Alon. The maximum number of Hamiltonian paths in tournaments. Combinatorica, 10(4):319-324, 1990.

[3] N. Alon. Problems and results in Extremal Combinatorics - III. Journal of Combinatorics, to appear.

[4] B. Bollobás. Extremal Graph Theory. Academic Press, 1978.

[5] A. Bondy. Basic graph theory: paths and circuits. In R.L. Graham, M. Grötschel, and L. Lovász, editors, Handbook of Combinatorics, volume 1, pages 3-110. North Holland, 1995.

[6] K. Corrádi and A. Hajnal. On the maximal number of independent circuits in a graph. Acta Mathematica Hungarica, 14(3-4):423-439, 1963.

[7] B. Cuckler. Hamiltonian cycles in regular tournaments. Combinatorics, Probability and Computing, 16(2):239-249, 2007.

[8] A. Ferber, M. Krivelevich, and B. Sudakov. Counting and packing Hamilton cycles in dense graphs and oriented graphs. arXiv preprint, arXiv:1212.4667, 2012.

[9] E. Friedgut and J. Kahn. On the number of Hamiltonian cycles in a tournament. Combinatorics, Probability and Computing, 14(5-6):769-781, 2005.

[10] T. Gustavsson. Decompositions of large graphs and digraphs with high minimum degree. PhD thesis, University of Stockholm, 1991.

[11] P. Keevash. The existence of designs. arXiv preprint, arXiv:1401.3665, 2014. 
[12] D. Kühn and D. Osthus. A survey on Hamilton cycles in directed graphs. European Journal of Combinatorics, 33(5):750-766, 2012.

[13] V. Rödl. On a packing and covering problem. European Journal of Combinatorics, 6(1):69-78, 1985.

[14] T. Szele. Kombinatorikai vizsgálatok az irányitott teljes gráffal kapcsolatban. Mat. Fiz. Lapok, $50(1943): 223-256,1943$.

[15] C. Thomassen. Hamilton circuits in regular tournaments. Annals of Discrete Math., 27:159$162,1985$.

[16] P. Turán. On an extremal problem in graph theory (in Hungarian). Mat. Fiz. Lapok, 48:436$452,1941$.

[17] R.M. Wilson. Decomposition of complete graphs into subgraphs isomorphic to a given graph. Congressus Numerantium, XV:647-659, 1975.

[18] N.C. Wormald. Tournaments with many Hamilton cycles. manuscript. 